\title{
Re: Effect of progestogen for women with threatened miscarriage: a systematic review and meta-analysis. (First comment on BJOG-19-1550.R1)
}

Adam Devall ${ }^{1}$, Ioannis Gallos ${ }^{1}$, Yakoub Khalaf ${ }^{2}$, Ben $\mathrm{Mol}^{3}$, Jackie Ross ${ }^{4}$, Andrew Shennan ${ }^{5}$, Andrew Horne ${ }^{6}$, Rachel Small ${ }^{7}$, Mariëtte Goddijn ${ }^{8}$, Madelon van Wely ${ }^{8}$, Mary Stephenson $^{9}$, Ole Bjarne Christiansen ${ }^{10}$, Jan Brosens ${ }^{11}$, Phillip Bennett ${ }^{12}$, Raj Raj ${ }^{13}$, Lesley Regan ${ }^{13}$, Siobhan Quenby ${ }^{11}$, and Arri Coomarasamy ${ }^{1}$

${ }^{1}$ University of Birmingham

${ }^{2}$ Guy's and St.Thomas NHS foundation trust

${ }^{3}$ Monash University Medical Centre

${ }^{4}$ King's College Hospital

${ }^{5}$ Kings College London

${ }^{6}$ University of Edinburgh

${ }^{7}$ Heart of England NHS Foundation Trust

${ }^{8}$ University of Amsterdam

${ }^{9}$ University of Illinois at Chicago College of Medicine

${ }^{10}$ Aalborg Universitet

${ }^{11}$ Warwick Medical School

${ }^{12}$ Imperial College

${ }^{13}$ Imperial College London

May 22, 2020

Dear Editor,

We would like to comment on the systematic review by Li et al.(1)

The use of steroid hormones in the first trimester is a serious issue as organogenesis takes place at this time and therefore there is the possibility of harm from not only congenital anomalies, but also long-term, and even inter-generational effects. Anyone investigating the use of steroid hormones in the first trimester should remember the diethylstilbestrol legacy of devastating harm. Oestrogen $\left(\mathrm{C}_{18} \mathrm{H}_{24} \mathrm{O}_{2}\right)$ and diethylstilbestrol $\left(\mathrm{C}_{18} \mathrm{H}_{20} \mathrm{O}_{2}\right)$ have similar molecular composition, but their effects are poles apart. In this review, the authors have combined progesterone with progestogens; however they are not the same, in the same way that oestrogen and diethylstilbestrol are not the same. Vaginal micronized progesterone, which we used in our large and high-quality trials (the PROMISE (2) and PRISM (3) trials), has identical molecular structure to natural progesterone, but the other drugs included in this review do not (Table 1). We chose to study vaginal micronized progesterone, as it is identical in structure to natural progesterone, and the available evidence and expert opinion suggested that this is least likely to cause harm. It is important to note that there is evidence of potential harm from dydrogesterone, particularly congenital heart disease.(4)

The authors make a bold statement in the abstract about the effects of dydrogesterone on live birth rate. However, they don't fully address the weaknesses in the evidence. Therefore, we wish to highlight the 
significant deficiencies in the two trials that contributed live birth data that led to the assertion of beneficial effects from dydrogesterone. Both studies were single centre, open-label studies without placebo control. ElZibdeh et al did not randomise participants, but instead allocated patients to dydrogesterone on Saturdays, Mondays and Wednesdays, and to no treatment on Sundays, Tuesdays and Thursdays. The trial by Pandian RU was not just a single-centre, but also a single-author study, with insufficient details of the methods to assess its quality. Thus, the effectiveness evidence from these trials cannot be considered reliable.

Approximately 80\% (4038 of 5056) of the data used in this systematic review come from our PRISM trial.(3) The PRISM trial is a prospectively-registered, randomised, placebo-controlled, multi-centre trial conducted to the highest standards in the UK. The trial found a $3 \%$ increase in live birth rate, but with borderline statistical significance $(\mathrm{RR}, 1.03 ; 95 \% \mathrm{CI}, 1.00$ to $1.07 ; \mathrm{P}=0.08)$. A pre-specified subgroup analysis in women with the dual risk factors of current pregnancy bleeding and one or more previous miscarriages found a $5 \%$ increase in live birth rate ( $\mathrm{RR}, 1.09 ; 95 \% \mathrm{CI}, 1.03-1.15 ; \mathrm{P}=0.003)$. In those with three or more previous miscarriages, a $15 \%$ increase in live birth rate was observed (RR, $1.28 ; 95 \%$ CI, 1.08 to $1.51 ; \mathrm{P}=0.004) .(3,5)$ No short-term safety concerns were identified. Based on these data, our recommendation is to consider vaginal micronized progesterone for women with early pregnancy bleeding and one or more previous miscarriages. As for the role of dydrogesterone, we need not only high-quality, randomised trial evidence of its effects but also credible evidence of its safety. As dydrogesterone is a synthetic progesterone-like drug, i.e. a progestogen but

not progesterone, the burden of proof to demonstrate short- and long-term safety rests on those promoting this drug.

\section{Authors}

Adam J Devall, Tommy's National Centre for Miscarriage Research, Institute of Metabolism and Systems Research, University of Birmingham, UK

Ioannis D Gallos, Tommy's National Centre for Miscarriage Research, Institute of Metabolism and Systems Research, University of Birmingham, UK

Yakoub Khalaf, Department of Women and Children's Health, School of Life Course Sciences, Kings College, London, UK

Ben Willem J Mol, Department of Obstetrics and Gynaecology, Monash University, Clayton, Australia

Jackie Ross, Kings College Hospital, King's College Hospital NHS Foundation Trust, London, UK

Andrew Shennan, Department of Women and Children's Health, School of Life Course Sciences, Kings College, London, UK

Andrew Horne, MRC Centre for Reproductive Health, The Queen's Medical Research Institute, University of Edinburgh, UK

Rachel Small, Birmingham Heartlands Hospital, University Hospitals Birmingham NHS Foundation Trust, Birmingham, UK

Mariëtte Goddijn, Center for Reproductive Medicine, Amsterdam UMC, University of Amsterdam, The Netherlands

Madelon van Wely, Center for Reproductive Medicine, Amsterdam UMC, University of Amsterdam, The Netherlands

Mary D Stephenson, Department of Obstetrics and Gynecology, University of Illinois at Chicago, Chicago, USA

Ole B Christiansen, Centre for Recurrent Pregnancy Loss of Western Denmark, Department of Obstetrics and Gynaecology, Aalborg University Hospital, Aalborg, Denmark

Jan J Brosens, Tommy's National Centre for Miscarriage Research, University of Warwick, UK 
Phil Bennett, Tommy's National Centre for Miscarriage Research, Imperial College London, UK

Raj Rai, Tommy's National Centre for Miscarriage Research, Imperial College London, UK

Lesley Regan, Tommy's National Centre for Miscarriage Research, Imperial College London, UK

Siobhan Quenby, Tommy's National Centre for Miscarriage Research, University of Warwick, UK

Arri Coomarasamy, Tommy's National Centre for Miscarriage Research, Institute of Metabolism and Systems Research, University of Birmingham, UK

\section{References}

1. Li L, Zhang Y, Tan H, Bai Y, Fang F, Faramand A, et al. Effect of progestogen for women with threatened miscarriage: a systematic review and meta-analysis. BJOG: An International Journal of Obstetrics \& Gynaecology.n/a(n/a).

2. Coomarasamy A, Williams H, Truchanowicz E, Seed PT, Small R, Quenby S, et al. A Randomized Trial of Progesterone in Women with Recurrent Miscarriages. N Engl J Med. 2015;373(22):2141-8.

3. Coomarasamy A, Devall AJ, Cheed V, Harb H, Middleton LJ, Gallos ID, et al. A Randomized Trial of Progesterone in Women with Bleeding in Early Pregnancy. N Engl J Med. 2019;380(19):1815-24.

4. Zaqout M, Aslem E, Abuqamar M, Abughazza O, Panzer J, De Wolf D. The Impact of Oral Intake of Dydrogesterone on Fetal Heart Development During Early Pregnancy. Pediatr Cardiol. 2015;36(7):1483-8.

5. Coomarasamy A, Devall AJ, Brosens JJ, Quenby S, Stephenson MD, Sierra S, et al. Micronized vaginal progesterone to prevent miscarriage: a critical evaluation of randomized evidence. Am J Obstet Gynecol. 2020;S0002-9378(19)32762-0.

Table 1. Molecular structure of natural progesterone and progestogens used for the prevention of miscarriage

\begin{tabular}{ll}
\hline Drug & Chemical structure \\
\hline Natural progesterone & $\mathrm{C}_{21} \mathrm{H}_{30} \mathrm{O}_{2}$ \\
Micronized progesterone & $\mathrm{C}_{21} \mathrm{H}_{30} \mathrm{O}_{2}$ \\
Dydrogesterone & $\mathrm{C}_{21} \mathrm{H}_{28} \mathrm{O}_{2}$ \\
17-hydroxyprogesterone & $\mathrm{C}_{21} \mathrm{H}_{30} \mathrm{O}_{3}$ \\
\hline
\end{tabular}

